\title{
Research on Simulating Rumen System Automatic Control and PH Value Detection
}

\author{
Zheng Liang ${ }^{1, a}$ and Jiannan Yuan ${ }^{2, b}$ \\ ${ }^{1}$ No.77, Hanlin Road, Economic and Technological Development District, Jilin prov. \\ ${ }^{2}$ No.3278, Weixing Road, Changchun City, Jilin prov. \\ ajlnyedu@163.com, barm9linux@163.com
}

Keywords: PLC; The configuration; Simulation system; PH sensor

\begin{abstract}
Because of ruminants with rumen this unique digestive organ, and utilization of roughage has a strong procession, so how to make the feed utilization, that is the first to solve the problem with this design. The subject of this item is in Jilin agricultural university college of animal science of the artificial rumen continuous culture system in vitro based on the design of the electric control part, in order to make the artificial rumen continuous culture in vitro system of automation degree and precision. His system can complete simulation of ruminant stomach mixing function, makes the artificial detection in the process of digestion of feed digestion and absorption of nutrients, such as for future breeding method and feed nutrition to provide important basis thesis.
\end{abstract}

\section{Introduction}

In the design of analog device were introduced in this paper, the existence of dynamic performance indicators, in order to make users more accurate and convenient to use numerical simulation device reads the PH sensor, then with a good HMI interface. So in the design we need to introduce a good hardware, and transparent to the configuration screen. So in this design, we use the embedded touch-screen of omron and its configuration software, make the device in the process of simulation, the user can be more detailed and clear observed simulating rumen and its parameters, the index of the whole simulation system and dynamic data. After the construction of the HMI man-machine interface, making the fermentation tank PH value by PLC, in the configuration screen to display, and it has the function of data report and report to the police, if match with the printer, the dynamic parameter can be a variety of ways such as images or digital preserved, convenient to users because of oversight and ignores the dynamic parameters, call the previous report, make the experiment more accurate finished. [1], The Paper Mainly Purpose and Significance:

(1) Realization of multi point measurement of $\mathrm{pH}$ value in rumen system;

(2) Sensor measurement and error theory and data analysis;

(3) The design of the configuration project and the production of WINCE system;

(4) Sensor signal acquisition and data analysis. Image processing technology development present situation [2]

\section{The Working Principle of This System}

The design by the main control module of PLC and 12 fermentation tank connected sensors communicate software used by the human machine interface of embedded system wince and configuration software real-time display the $\mathrm{pH}$ value of the 12 in the fermentation tank, the main hardware circuit using EPLAN design, software combined with bubble method and mean filtering method to improve the precision processing method. 


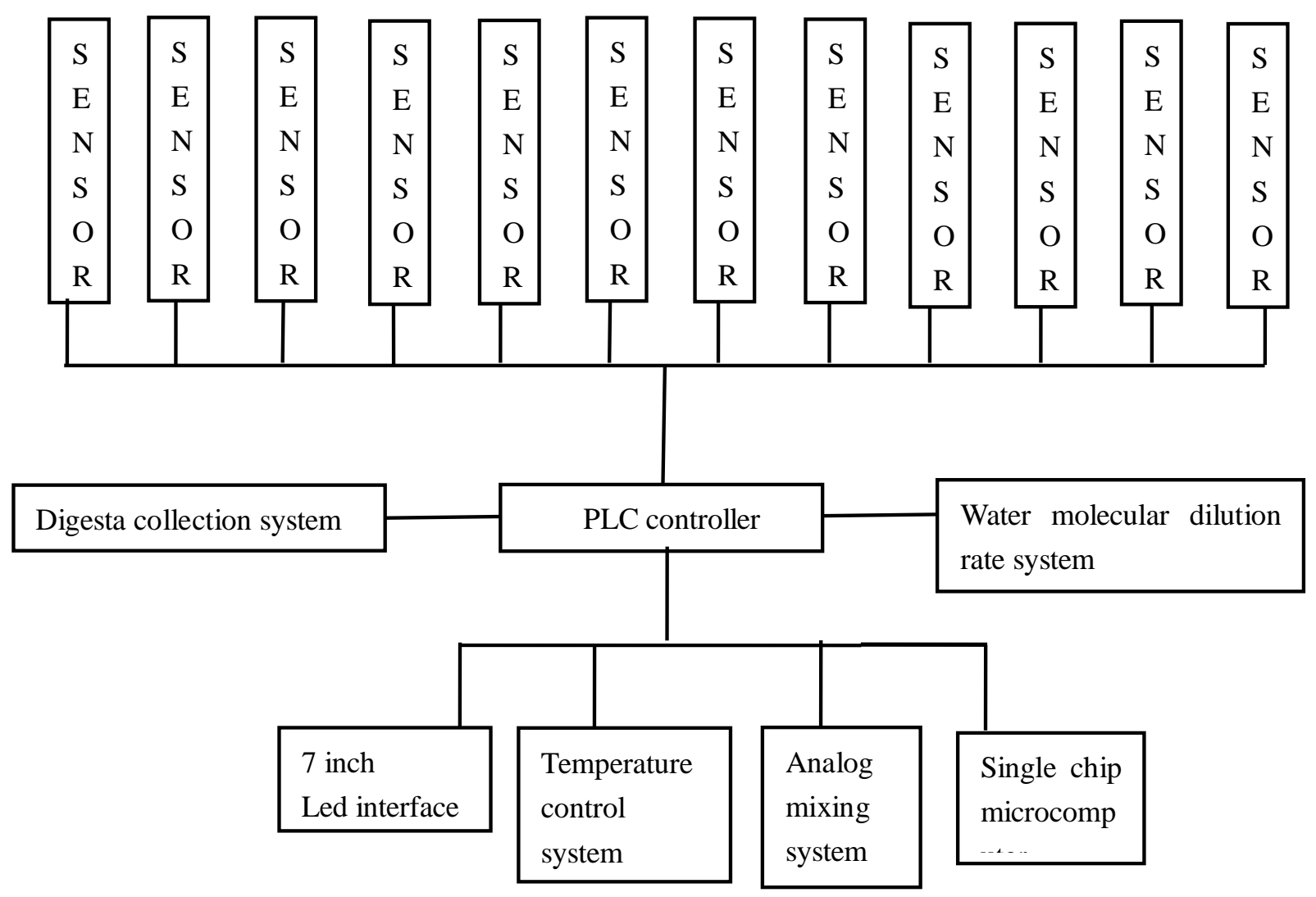

Figure 1. The method of PLC design method

\section{The Introduction of S7-2000 PLC}

S7--200 series CPU221--226 each have two types of relay and transistor CPU, to match the different power supply voltage and output voltage control.

(1) s7 --200PLC digital input / output point

(2) the machine analog input / output point

(3) the way of communication

(4) memory EEPROM module

(5) PLC power supply battery module
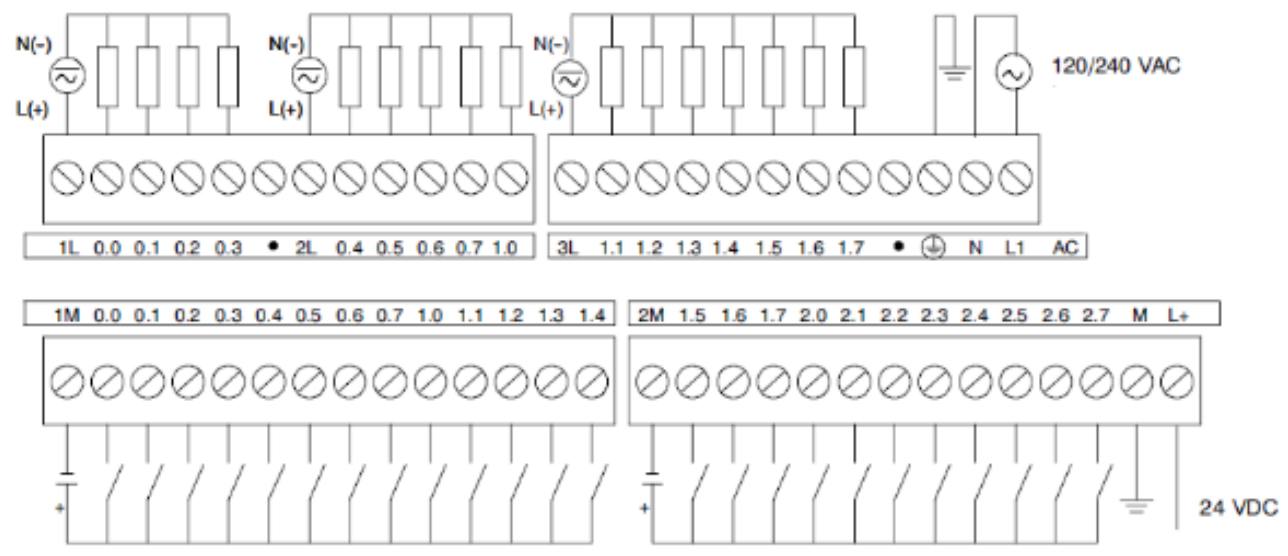

Figure 2. The method of PLC connection

\section{The EM 231 I/O Port Description}

Module EM231 upper, a total of 12 terminals, each of the three points for a group (such as re, a + , a) 
can be used as a road analog input channel, a total of four groups, for voltage signal using only two terminals, the current signal need three terminal, wherein $\mathrm{R}$ should be on the + side short circuit for unused input channel should be shorted. The lower part of the left M module and L+ ends should access DC24V power, the right to calibrate the potentiometer and DIP switch, terminal wiring diagram as follows[4]:

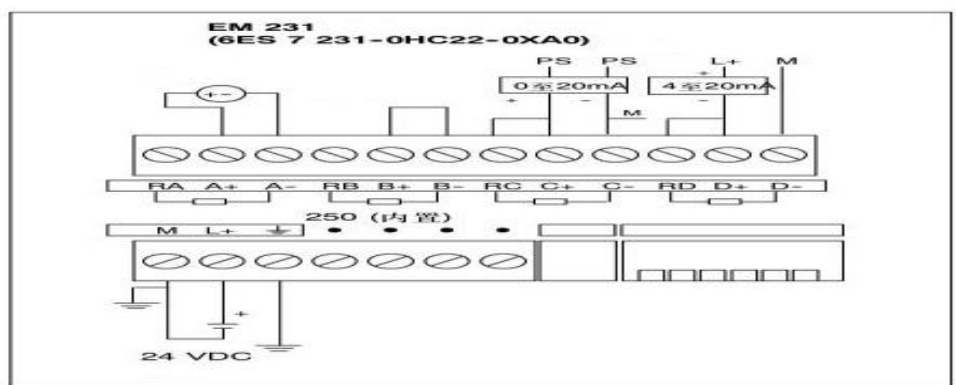

Figure 3. The method of EM 231 connection

\section{The Introduction of NTZ - Designer NT Link}

The basic function key of the software is "button, indicator light, graphics library [5]" and so on. It also has the function of "history curve, trend curve, real time alarm" and so on. Of course, each software has its own unique features, Designer - NT Link NTZ software with a variety of alarm settings, and there are online simulation functions, and provides a variety of macro functions, to facilitate the user's programming [6]. The communication interface diagram as shown in Fig. 4.
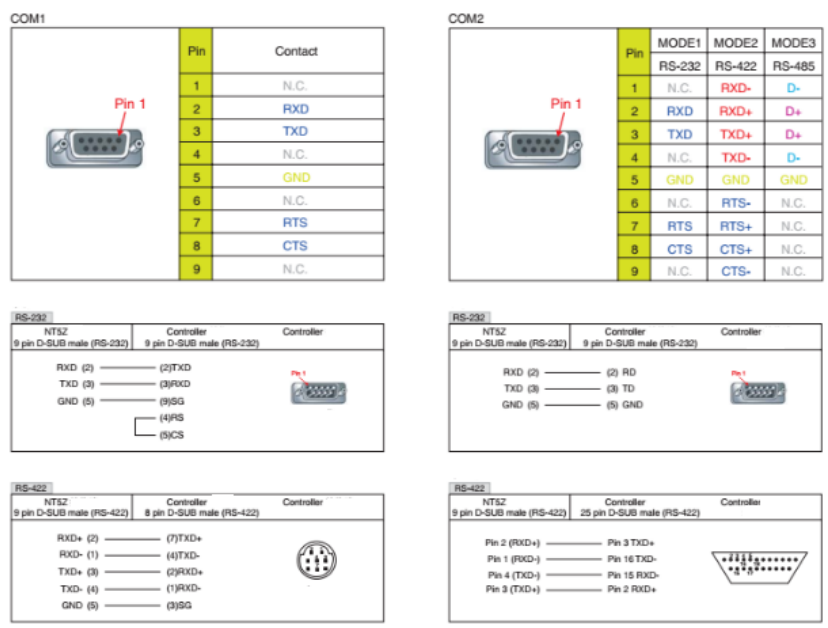

Figure 4. NTZ - Designer connection

\section{Analysis of Experimental Results}

From the chart we can see our power by civil $220 \mathrm{~V}$ AC power supply, leads to the ends are known as $1, \mathrm{n}$ two power lines, the zero line and FireWire and after knife switch to ensure can at any time for manual power outages [7]. The second step is to access the fuse, if below circuit overload or short circuit, the fuse can be automatically fuse, if conditional can join can automatically fix the fuse, so you can better the protection circuit [8], and connected to the air switch can not only control the manual off section of the circuit, but also can fuse and the circuit to double insurance and dual closed off. And then access the $220 \mathrm{~V}$ AC to $24 \mathrm{~V}$ DC switching power supply, the circuit of the transformer and rectifier[9]. The four lines are respectively connected with an air switch to ensure that each line can be controlled to ensure the safety of the equipment [10] 
Figure 5-7. Zoom in two times-four times.

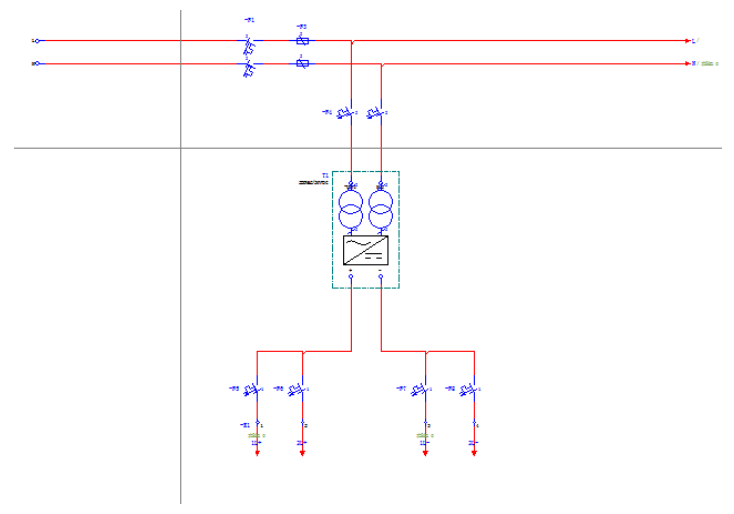

Figure 5. Primary circuit

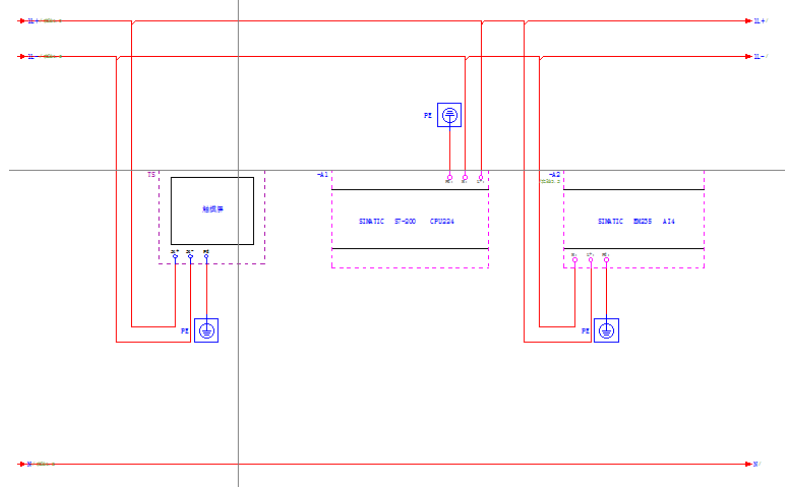

Figure 6. Power circuit

\section{Acknowledgements}

The 199th [2016] No.of JI Jiao Ke He Zi

The 221th [2015] No.of JI Nong Yuan He Zi

\section{References}

[1] Li P, Lehman B. Performance prediction of DC-DC converters with impedancesas loads [J].IEEE transactions on power Electronics, 2014, 19(1):201-208.

[2] Johnson Ronald R.Symposium on microbial digestion in ruminant: in vitro rumen fermentation techniques [J] J. Animal S ci, 1963, 22: 792-800. Cha lu pa, 1977

[3] Cone,J.W., A.H.Van gelder,et al. Different Techniques to Study Rumen Fermentation Characteristics of Maturing Grass and Grass Silage, J Dairy Sci,1999,82:957-966.

[4] Men ke K H, Raab L, Salewski A. The estimation of the digestibility and metabolisable energy content of ruminant feeding stuffs from the gas production when they are incubated with rumen liquor [J].Journal of Agricultural Science, 1979, 93:217-222.

[5] Tilley J M A, Terry R A.A two-stage technique for the digestion of forage crops [J]. Journal of the British Grassland Society,1963,18:104-111. Goering, Van Soest, 1970.

[6] Warner, A.C.I. Criteria for establish the validity of in vitro studies with rumen microorganism in so called artificial rumen system.J.Gen.Microbiol.1956, 14:733-739.

[7] P.G. Clem, M. Rodriguez, J.A. Voigt and C.S. Ashley, U.S. Patent 6,231,666. (2001) Information on http://www.weld.labs.gov.cn 